\title{
Laser-induced carbon pyrolysis of electrodes for neural interface systems
}

\author{
Ana Oliveira (1), Juan S. Ordonez (2,3), Danesh Ashouri Vajari (1), Max \\ Eickenscheidt (1), Thomas Stieglitz (1)
}

(1) Laboratory for Biomedical Microtechnology, Department of Microsystems Engineering, University of Freiburg, Germany; (2) Medtronic Eindhoven Design Center (MEDC), Eindhoven, The Netherlands; (3) Freiburg Institute of Advanced Studies (FRIAS), Freiburg, Germany

This article is distributed under the terms of the Creative Commons Attribution Noncommercial License (CC BY-NC 4.0) which permits any noncommercial use, distribution, and reproduction in any medium, provided the original author(s) and source are credited.

\begin{abstract}
The objective of this work is to produce a laser-fabricated polymer-metal-polymer electrode with the merit of a carbon-based coating as the active site. A $10 \mu \mathrm{m}$-thick layer of parylene-C is used serving as the insulation layer in which the active site is locally laser-pyrolyzed. Our preliminary results show that the proposed method is promising in terms of fabrication feasibility and desired electrochemical capabilities.
\end{abstract}

Key Words: Laser-induced carbon pyrolysis, electrodes, neural interface systems

Eur J Transl Myol 2016; 26 (3): 181-186

Neural interfacing systems (NIS) can act as a bridge between the body and the exterior world when the communication between the brain and the muscles or organs is compromised. ${ }^{1}$ Electrodes are known as one of the most crucial part of the NIS due to their functionality as an active interface in direct contact with the tissue. Noble metals have been widely used as electrode material for both stimulation and recording applications. ${ }^{2,3}$ However, degradation mechanisms, for example due to limited charge injection capacity restrict their usage. ${ }^{4,5}$ Miniaturized interfaces, on which the geometrical surface area for a contact is restricted, challenge the development of new materials with a high electrochemical performance. Lately, carbon based materials have emerged as a versatile and potential substitutes for metal electrodes. ${ }^{6}$ This is attributed to their superior properties such as biocompatibility, chemical inertness, mechanical robustness and good electrochemical properties due to a large effective surface area of contact. ${ }^{4,5,7}$ Amongst carbon materials, glassy carbon and Q-carbon appears as very promising materials. ${ }^{8,9}$ Glassy carbon has already showed it's applications in neural prosthetics, with unique electrochemical performance. ${ }^{3,8}$ The just recently discovered Q-carbon appeared to be a very promising material by facilitating improved properties such as mechanical hardness, electrical conductivity, chemical and physical characteristics. ${ }^{9}$ Q-carbon is produced using a laser and having an amorphous carbon coating as the initial substrate. ${ }^{9}$ Glassy carbon for instance can be produced by the pyrolysis of high- carbon content polymers such as Kapton ${ }^{10}$ and Su- $8 .^{3}$ The typical pyrolysis process consists in using a furnace at high temperatures in an inert atmosphere. ${ }^{7}$ This high temperature restricts the fabrication procedure, as it has to take place at the beginning of the microfabrication procedure, unless the electrodes are composed of purely semiconductor or ceramic materials. Photolithography technique can be also employed ${ }^{7}$ but it requires the use of masks and clean room facilities which raises the complexity and fabrication costs. However, using the laser as pyrolysis method has also been reported. ${ }^{7,10}$ The pyrolysis method using laser is advantageous as compared to the other mentioned methods as it avoids the use of high temperatures and requires no need for the cleanroom processes. Laser technology enables local carbonization at the desired spot, is fast and allows highest flexibility. ${ }^{11}$ Within this work, we report on a novel electrode fabrication process that merges the cost, time and production advantages of multiple technological processes to fabricate electrodes with advanced electrochemical performance. The goal of this work is also to investigate the properties of laser- based carbon pyrolysis for electrodes in NIS and bring its development closer to in vivo applications. The proposed method uses laser-machining to produce structured polymer-metal-polymer electrode arrays. The elegance of the process lies in the fact that the same material serving as a coating and insulation between metal lines is locally transformed by laser to an improved carbon- 


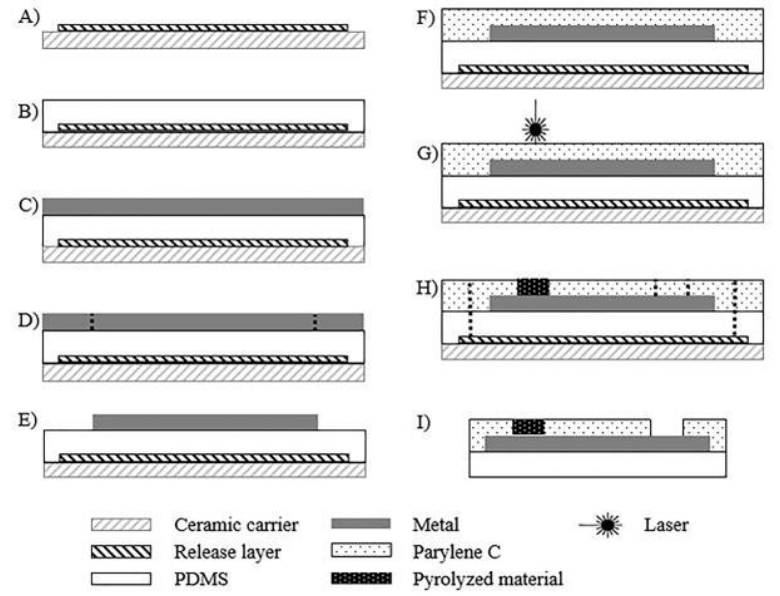

Fig 1. Cross-section schematic of the array process fabrication: A) lamination of the release layer; $B)$ spin coat PDMS; C) lamination of the metal; $D)$ laser structuring of the metal; E) removal of metal excess; F) parylene- $C$ deposition; $G$ ) laser pyrolysis; $H$ ) contact opening and cut of the electrode borders; I) electrode peel off.

based coatings at the active sites. The material used as precursor of the carbon-based material is parylene-C which is known as a biocompatible, mechanically robust and medically approved material. ${ }^{2}$

\section{Materials and Methods}

\section{Process}

The fabrication process starts with lamination of selfadhesive tape (No. 4124 by Tesa AG, Hamburg, Germany) onto a ceramic carrier (Fig.1.A). This sheet acts as release layer to separate the electrode from the ceramic carrier in the last step. Then, a layer of approximately $200 \mu \mathrm{m}$ diluted silicone rubber (MED1000,NuSil, Carpinteria, USA) was spin coated on top (Fig.1.B). The diluted silicone rubber (with n-heptane in a volume ratio of $1: 1$ ) was used in order to yield a more homogeneous coating. Before the spin coated silicone rubber is fully cured, a thin platinum-iridium foil (25 $\mu \mathrm{m})$ was laminated onto it (Fig.1.C). A nanosecond pulsed laser (DPL Genesis Marker Nd:YAG, ACI Laser $\mathrm{GmbH}$, Deutschland) was used to cut out the electrode sides, tracks and contacts (Fig.1.D). In order to anchor the foil and the polymers, additional small openings were introduced in the outer vicinity of the electrode active site. ${ }^{2}$ Having removed the excess metal with tweezers, a parylene-C (DPXC by Specialty Coating Systems, Indianapolis, USA) layer of $10 \mu \mathrm{m}$ is deposited on top (Fig1.E-F) with a parylene-C deposition unit (PDS 2010 LAB-COTERTM by Specialty Coating Systems, Indianapolis, USA). This polymer has a high quantity of carbon in composition which makes it suitable to be a precursor of the carbonized material. Using the same laser, but different parameters, the parylene-C was carbonized by pyrolysis at the desired site on top of the platinum layer. Here, the pyrolyzed material forms a

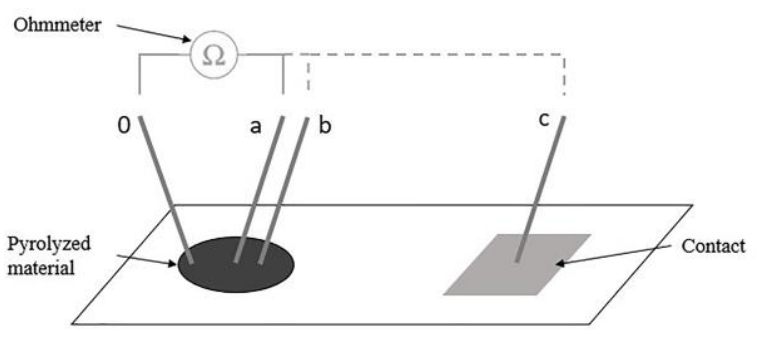

Fig 2. Setup used to achieve a conductance approximation. Electrode active site (left) and contact pad (right) are connected via an insulated metal line under the top layer (not shown in the graph).

conductive connection to the platinum-iridium foil (Fig.1.G). Laser ablation was used, to remove the Parylene- $\mathrm{C}$ at the distal end from the interconnection contact pad and also to cut the perimeter of the array. Reference electrodes with no carbon coating were produced by cutting the perimeter of the electrode site and peeling off excess material from the surface. Lastly, the electrode was peeled off the release layer. (Fig.1.HI). The used laser was an infrared nanosecond (pulse width of 30 to $90 \mathrm{~ns}$ ) with wavelength of $1064 \mathrm{~nm}$. Therefore the interaction with the materials was predominantly of thermal nature. ${ }^{12}$ In order to avoid burning of the material, all experiments were performed in saturated $\mathrm{N}_{2}$ environment. The usage of laser causes the material to reach a certain temperature in which volatile elements are removed, new $\mathrm{C}-\mathrm{C}$ bonds are formed and hence, the polymer pyrolysis can be realized. ${ }^{6}$ Since the plasma temperature at the laser focus point ( $35 \mu \mathrm{m}$ in diameter) is influenced by the material characteristics, laser parameters and environmental conditions, ${ }^{13}$ a scope of laser parameters to generate carbon electrodes was studied. The laser parameters have especially high influence on the power density reaching the sample and therefore in the generation of the desired material. ${ }^{9}$ The best combination of laser parameters i.e. power, frequency, speed and pulse duration, was used to produce an electrode active site of $700 \mu \mathrm{m}$ in diameter. All materials used to fabricate the samples were biocompatible and medical grade.

\section{Composition of electrodes}

Composition analysis of the carbonized electrodes was done using X-ray photoelectron spectroscopy (XPS). The measurements were performed using a K-Alpha+ XPS spectrometer (Thermo Fisher Scientific, East Grinstead, UK). Data acquisition and processing using the Thermo Avantage software is described elsewhere. ${ }^{14}$ The sample was analyzed using a microfocused, monochromated Al K $\alpha$ X-ray source (30-400 $\mu \mathrm{m}$ spot size). The spectra were fitted with one or more Voigt profiles (binding energy uncertainty: $+/-0.2 \mathrm{eV}$ ). The analyzer transmission function, Scofield sensitivity 


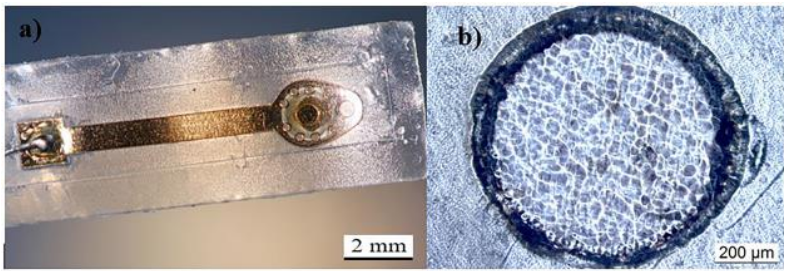

Fig 3. Picture of the produced electrode (A) and micrograph showing the electrode active site $(B)$.

factors ${ }^{15}$ and effective attenuation lengths (EALs) for photoelectrons were applied for quantification. EALs were calculated using the standard TPP-2M formalism. ${ }^{16}$ All spectra were referenced to the $\mathrm{C} 1 \mathrm{~s}$ peak of hydrocarbon at $285.0 \mathrm{eV}$ binding energy controlled by means of the well-known photoelectron peaks of metallic $\mathrm{Cu}, \mathrm{Ag}$, and $\mathrm{Au}$. Prior to spectral measurements the samples were sputter cleaned using an Ar1000+ cluster ion beam at $8 \mathrm{keV}$ and $30^{\circ}$ angle of incidence which did not harm the arrays.

\section{Conductance Test}

In order to have an estimation of the conductance $(\sigma)$ of the pyrolyzed material the resistance $(\rho)$ was measure according to Eq.1.

$$
\sigma=\frac{1}{\rho}
$$

The measurements $(n=10)$ were taken at different positions ( $\mathrm{a}, \mathrm{b}$ and $\mathrm{c}$ ) versus an origin point (0) using an ohmmeter (34401A 6 1/2 Digit Multimeter, Agilent) with two microneedles (see Fig.2). Special care was taken when placing the needles to ensure minimal scratching of the surface. The distance between the origin point and the $\mathrm{a}, \mathrm{b}$ and $\mathrm{c}$ point was approximately 0.3, 0.6 and $10 \mathrm{~mm}$, respectively. Here $\mathrm{a}$ and $\mathrm{b}$ were located inside the pyrolyzed area and point $\mathrm{c}$ was found at the electrode contact.

\section{Electrochemical Characterization}

Electrochemical characterization of the fabricated electrodes was addressed using electrochemical impedance spectroscopy (EIS) and cyclic voltammetry (CV). Measurements were performed in a three electrode setup with a platinum counter electrode and $\mathrm{Ag} / \mathrm{AgCl}$ reference electrode. Both $\mathrm{CV}$ and EIS were conducted in $0.9 \%$ saline solution. Electrochemical interface was provided by a potentiostat and a frequency analyzer device (Solartron 1260\&1287 by Solartron Analytical, Farnborough, Hampshire, UK) accompanied by the software Zplot (v2.8 by Scribner Associates Inc., Southern Pines, NC, USA) to control the setup, and save and analyze the data. The EIS measurements were made within the frequency range of $0.1 \mathrm{~Hz}-1 \mathrm{MHz}$ with an excitation amplitude of $0.5 \mathrm{mV}$. In order to run $\mathrm{CV}$ measurements the vertex potentials were set between -

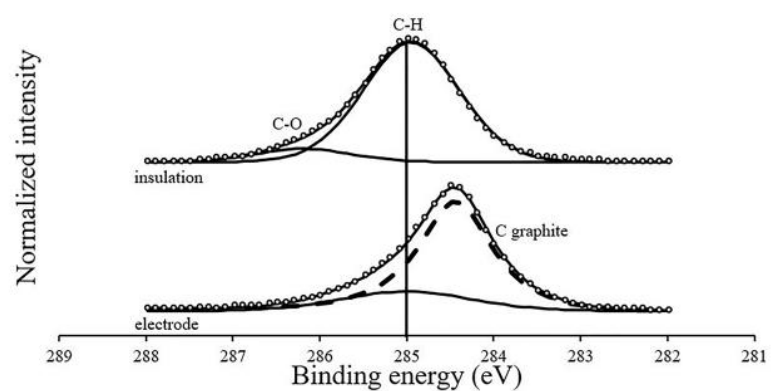

Fig 4. Detail XPS carbon spectra $(C 1 s) X P$ of the electrode material (bottom) and its raw material (insulator, top). For a better visualization the spectra are normalized to maximum intensities. The peak shape definition as well as the peak assignments are according to ${ }^{17}$

$0.6 \mathrm{mV}$ and $0.8 \mathrm{mV}$ and the working electrode potential was swept at a scan rate of $50 \mathrm{mV} / \mathrm{s}$.

\section{Pulse Test}

Pulse test (voltage transient measurement) was used to study the maximum polarization, both most negative $\left(E_{\mathrm{mc}}\right)$ and most positive $\left(\mathrm{E}_{\mathrm{ma}}\right)$, of the electrodeelectrolyte interface under a current-controlled stimulation pulse. This test was conducted in phosphate buffered saline (PBS). The used current pulse was a rectangular shaped, symmetrical, biphasic, charge balanced, negative phase first waveform. The amplitude was set at $200 \mu \mathrm{A}$ (pulse width of $200 \mu$ s and the pulse repetition frequency of $20 \mathrm{~Hz}$ ).

\section{Results}

\section{Process}

In this work samples with simple structure designs were produced as can be seen in (Fig.3.A). Is also notable that the using of polymers provides flexibility, not compromising its robustness. Through optical inspection with microscope (LEICA DM4000M), the surface structure of the pyrolyzed active site shows to be rough (Fig.3.B).

\section{Composition of electrodes}

One sample was analyzed with XPS to reveal the chemical and elemental composition of the original insulation layer (parylene-C) and the pyrolyzed electrode. The dominating atomic bonds in parylene-C are $78.3 \%$ carbon-hydrogen $(\mathrm{C}-\mathrm{H}, \mathrm{C} 1 \mathrm{~s}=285.0 \mathrm{eV})$ and $8.7 \%$ carbon-oxygen $(\mathrm{C}-\mathrm{O}, \mathrm{C} 1 \mathrm{~s}=286.8 \mathrm{eV})$. There are no indicators for graphitic carbon or for the underlying platinum, nor high concentrations of salts. In contrast, the carbonized area consist mainly of graphitic carbon at $\mathrm{C} 1 \mathrm{~s}=284.4 \mathrm{eV}$ (51.5\%), some C-H leftovers (13.4\%) and a high amount of sodium (13.4\%) and chloride $(13.0 \%)$. Beside other minor contaminations, little platinum $(<2 \%)$ is exposed on the electrode surface. 


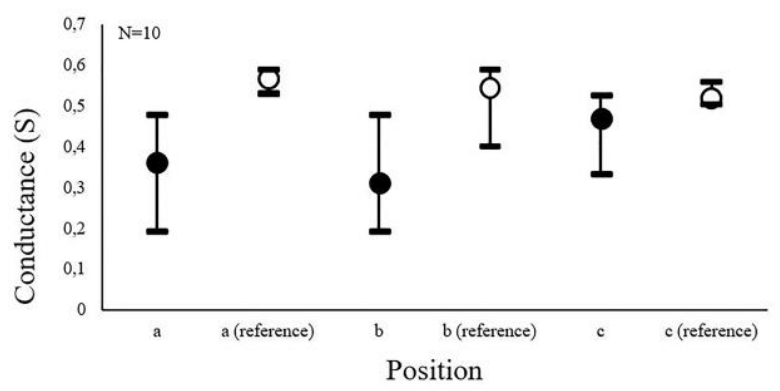

Fig 5. Conductance measurements of the pyrolyzed material and of a Pt:Ir reference at the different position shown in Figure 2. Data show mean, minimum and maximum values.

\section{Conductance Test}

The pyrolyzed material is conductive, presenting a mean conductance at position $\mathrm{a}$ and $\mathrm{b}$ of about $0.4 \mathrm{~S}$ and 0.3 $\mathrm{S}$, respectively (Fig. 5, black markers). Furthermore, a conductive transition between the pyrolyzed layer and the metal layer can be verified from the values measured at position c. The conductivity for Pt:Ir was measured slightly higher (approx. 0.5 S, Fig.5 white markers).

\section{Electrochemical Characterization}

To study the electrode performance under stress conditions, some electrodes were cycled for a longer time frame. i.e. 90 cycles at scan rate of $200 \mathrm{mV} / \mathrm{s}$. The impedance magnitude of the pyrolyzed electrode was found to be about $55 \mathrm{~K} \Omega$ higher than the measured impedance for to the reference (see Fig.6). The impedance behavior of the pyrolyzed samples remained approximately the same after 70 cycles of the cyclic voltammetric stress (Fig.7) but showed a decrease after 90 cycles.

The obtained cyclic voltammograms revealed an increase in the charge storage capacity (CSC) in the pyrolyzed electrodes after 70 cycles of voltammetric stress (Fig.7). It is also noticed that the voltammogram shape of the pyrolyzed material differs from the reference including a wider water window (Fig.8).

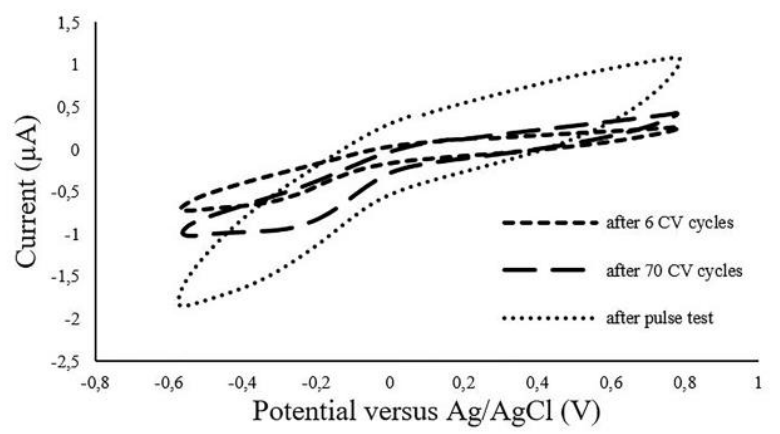

Fig 7. Voltammogram of pyrolyzed electrode at beginning (after $6 \mathrm{CV}$ cycles), after $70 \mathrm{CV}$ cycles and after pulse test.

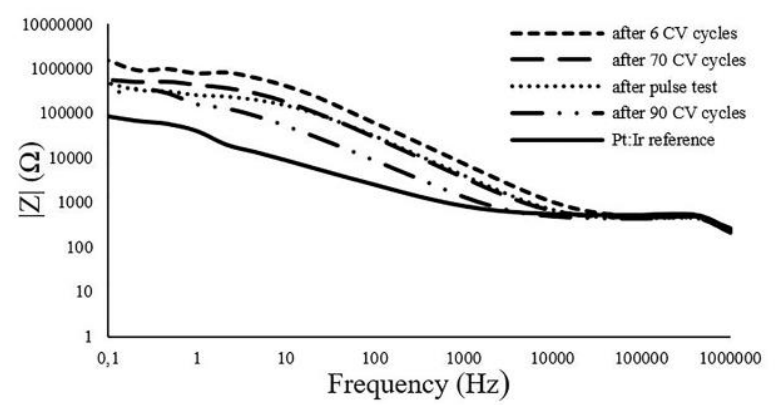

Fig 6. EIS measurements of the Pt:Ir electrode and of the pyrolyzed electrode on beginning (after $6 \mathrm{CV}$ cycles), after $70 \mathrm{CV}$ cycles, pulse test and $90 \mathrm{CV}$ cycles.

\section{Pulse Test}

The injected charge density obtained from the used waveform corresponds to $10.3 \mu \mathrm{C} / \mathrm{cm}_{2}$, calculated using Eq.2.

$$
Q=\frac{I . t}{A}
$$

Where $\mathrm{Q}(\mu \mathrm{C} / \mathrm{cm} 2)$ is the charge density, I is the applied current $(\mathrm{A}), \mathrm{t}$ is the pulse width $(\mu \mathrm{s})$ and $\mathrm{A}(\mathrm{cm} 2)$ is the geometric surface area of the electrode.

The measured potential drop across the access resistance was found to be roughly the same for both the pyrolyzed electrode and the Pt:Ir reference, corresponding approximately $100 \mathrm{mV}$ (Fig.9, initial step).

The pulse test, as described in method section resulted in $E_{\mathrm{mc}}$ of, approximately $1 \mathrm{~V}$ in case of pyrolyzed electrodes which was about $700 \mathrm{mV}$ higher than the measured value for the reference Pt-Ir (Fig.9). However, after stressing the electrode by cyclic voltammetry the $\mathrm{E}_{\mathrm{mc}}$ experienced a drop of, approximately, $300 \mathrm{mV}$.

The voltage excursion observed for the pyrolyzed material remained within the water window limit throughout the measurement even in case of ramping up the injected current up to $1000 \mu \mathrm{A}$.

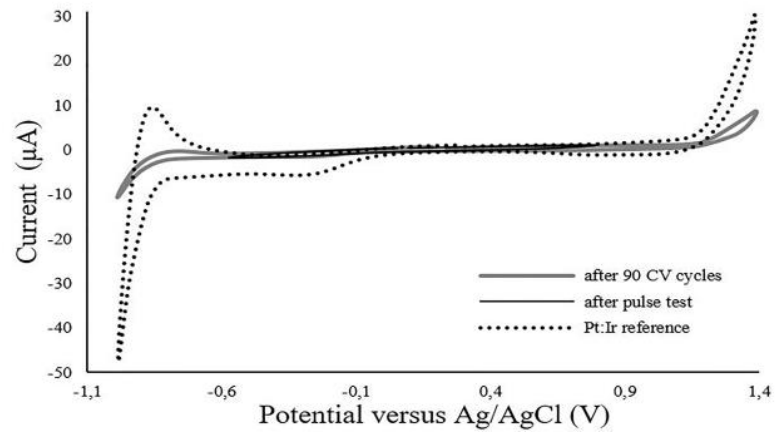

Fig 8. Voltammogram of the pyrolyzed electrode after pulse test and after $90 \mathrm{CV}$ cycles and of the Pt:Ir reference. 


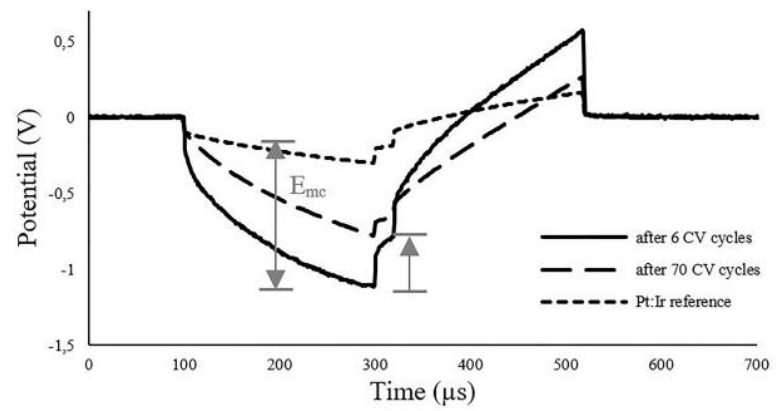

Fig 9. Voltage responses of the pyrolyzed material without stress exposure (after 6 CV cycles), after $70 \mathrm{CV}$ cycles and of the Pt:Ir reference electrode to $200 \mu \mathrm{A}, 200 \mu \mathrm{s}$, biphasic pulses.

\section{Discussion}

The image taken of the pyrolyzed active site revealed a rough surface which could suggest a high charge injection storage capacitance. However, further investigations are required in order to draw a concrete conclusion on that. Furthermore, a smooth transition between insulation and the active site was observed which can be advantageous with respect to the concerns on the tissue interactions. XPS data showed a clear transformation of the chemical structure of parylene-C to a graphitic carbon layer. Since sodium-chloride is not present in the fabrication process, it is most likely a left over contamination from the electrochemical tests performed prior to the XPS measurements. The obtained cyclic voltammograms reveal change in the electrochemical performance of the pyrolyzed electrode as compared to the Pt-Ir reference. The observed characteristics can suggest the possible presence of carbon substances, which is in agreement with the XPS results. Observed in the electrode cycled for 70 cycles, a plausible surface activation can be suggested in case of the pyrolyzed electrodes. This can be attributed to a surface activation under the stress condition resulted from the slow kinetic transform. The observed behavior can also explain the smaller voltage excursion of the pyrolyzed samples after $70 \mathrm{CV}$-cycles as compared to the freshly pyrolyzed electrodes. The surface activation can be due to the presence of residual parylene-C on the surface, preventing a full access of the pyrolyzed active site. Repetitive CVs can potentially sweep the residues off the surface. This in turn would also result in a more pronounced carbon-featured surface and hence, supports the proposed concept. However, further studies to investigate the performance of the electrode under higher charge injection levels should be conducted.

Despite of a lower performance of the produced electrodes as compared to the given characteristics for the Pt-Ir reference, the obtained results can suggest that an improvement in the electrochemical performance of the novel carbon-based electrode is possible.

Furthermore, the feasibility of the proposed methodology regarding simplicity, time and cost efficiency was successfully addressed. This proposed methodology addressing the carbon-based electrode can potentially provide the field of research with a versatility of applications, e.g. neurotransmitter detection, and hence, would pave the way for advancing the available technologies. The current method was demonstrated on a foil- based low cost technology. Nevertheless, thin-film fabricated devices, e.g. flexible polyimide-based and parylene substrates as well as the rigid silicon devices, can also benefit from this engineered combination of techniques. The used laser spot size is of $35 \mu \mathrm{m}$ in diameter, and could be reduced with the appropriate optics. Nevertheless, until optimal material conditions have been detected, the presented method remains an accessible way to further study the local pyrolysis of carbon into a desired composition for the target application. The proof of concept was provided. However, more investigation is yet needed in order to obtain a better understanding of the surface characteristics and also to address the biocompatibility and biostability concerns.

\section{Acknowledgments}

The authors would like to thank M. Bruns from Karlsruhe Nano Micro Facility (KNMF), a Helmholtz research infrastructure at KIT Karlsruhe Institute of Technology for the XPS measurements. The used KAlpha+ instrument was financially supported by the Federal Ministry of Economics and Technology on the basis of a decision by the German Bundestag.

The authors would also like to thank the BrainLinks BrainTools Cluster of Excellence (German Research Foundation DFG, EXC 1086) for funding the research.

\section{Corresponding Author}

Prof. Thomas Stieglitz, Laboratory for Biomedical Microtechnology, Department of Microsystems Engineering, University of Freiburg, Germany.

Email: stieglitz@imtek.uni-freiburg.de

Email of CoAuthors

Ana Oliveira: ana.oliveira@uranus.uni-freiburg.de Juan Ordonez: juan.ordonez.orellana@medtronic.com Danesh Ashouri Vajari: danesh.ashouri@imtek.unifreiburg.de

Max Eickenscheidt: max.eickenscheidt@imtek.unifreiburg.de

\section{References}

1. Hatsopoulos N, Donoghue J. The science of neural interface systems. Annu Rev Neurosci 2009;32:249-66.

2. Ordonez J, Schuettler M, Boehler C, Boretius T, Stieglitz T. Thin films and microelectrode arrays for neuroprosthetics. MRS Bull 2012;37:590-8.

3. Kassegne $\mathrm{S}$, Vomero $\mathrm{M}$, Gavuglio $\mathrm{R}$, et al. Electrical impedance, electrochemistry, mechanical stiffness, and hardness tunability in 


\section{Laser-induced carbon pyrolysis of electrodes for neural interface systems}

Eur J Transl Myol 26 (3): 181-186

glassy carbon MEMS eCoG electrodes.Microelectron Eng 2015;133:36-44.

4. Vitale F, Summerson B, Aazhang SR, et al. Neural Stimulation and Recording with Bidirectional, Soft Carbon Nanotube Fiber Microelectrodes. ACS Nano 2015;9:4465-74.

5. Cogan SF. Neural stimulation and recording electrodes. Annu Rev Biomed Eng 10, pp. 275309, 2008.

6. Madou M, Sharma S. Micro and nano patterning of carbon electrodes for bioMEMS Bioinspired Biomim Nanobiomaterials. 2012;1:252-65.

7. McCreery RL. Advanced carbon electrode materials for molecular electrochemistry. Chem Rev 2008;108:2646-87.

8. Dekanski A, Stevanović J, Stevanović R,et al. Glassy carbon electrodes. Carbon NY 2001;39:1195-1205.

9. Narayan J, Bhaumik A. Novel phase of carbon, ferromagnetism and conversion into diamond. J Appl Phys 2015;118:1-10.

10. Wynn GH, Iii AWF. Development and Characterization of Electrochemical Devices Using Ultraviolet Laser Induced Carbonization of Polyimide Films. J Electrochem Soc 1997;144:3769-72.

11. Suaning GJ, Schuettler M, Ordonez JS, Lovell NH. Fabrication of multi-layer, high-density microelectrode arrays for neural stimulation and bio- signal recording, Proc. 3rd Int. IEEE EMBS Conf Neural Eng 2007, 5-8.

12. Kohler F, Schuettler M, Ordonez J, Stieglitz T. Laser Microfabrication of Neural Electrode Arrays: Comparison of Nanosecond and Picosecond Laser Technology. Proc IFESS 2011;102:99-101.

13. Mortazavi SZ, Parvin P, Mousavi Pour MR, et al. Time-resolved evolution of metal plasma induced by Q-switched Nd:YAG and ArF-excimer lasers. Opt Laser Technol 2014;62:32-9.

14. Parry KL, Shard A, Short R, et al. RXPS characterisation of plasma polymerised surface chemical gradients. Surf Interface Anal 2006;38:1497-1504.

15. Scofield JH. Hartree-Slater subshell photoionization cross-sections at 1254 and 1487 eV. J Electron Spectros Relat Phenomena 1976;8:129-37.

16. Tanuma S, Powell CJ, Penn P. Calculations of electron inelastic mean free paths. V. Data for 14 organic compounds over the 50-2000 eV range. Surf Interface Anal 1994;21:165-76.

17. Zydziak N, Christof H, Bruns M., BarnerKowollik C. One-Step Functionalization of SingleWalled Carbon Nanotubes ( SWCNTs ) with Cyclopentadienyl-Capped Macromolecules via Diels À Alder Chemistry. 2011;44:3374-338. 